\title{
Left inferior accessory renal arteries its embryological basis and clinical significance
}

\author{
N. Satyanarayana ${ }^{1}$, R. Guha ${ }^{2}$, V. Nitin ${ }^{3}$, G. Praveen ${ }^{4}$, A.K. Datta ${ }^{5}$, P.Sunitha ${ }^{6}$ \\ ${ }^{1}$ Assistant Professor , ${ }^{2}$ Professor,${ }^{3,4}$ Lecturer , ${ }^{5}$ Professor and Head Dept. of Anatomy, ${ }^{6}$ Assistant Professor, Physiology, College \\ of Medical Sciences, Bharatpur, Nepal.
}

\begin{abstract}
The variations in origin of renal arteries are very common. During routine dissection of a male cadaver we found left accessory renal artery. The origin of accessory renal artery is immediately below main renal artery. The left inferior accessory renal arteries run towards inferior pole of left kidney, in the area where the left renal vein was leaving the left kidney. The vascular variations are very important for vascular surgeons, urologists, nephrologists, radiologists.
\end{abstract}

Key Words: Renal artery, accessory renal artery, renal vein.

\section{Introduction}

The renal arteries are two largest branches of abdominal aorta and arise laterally, just below the origin of the superior mesenteric artery. The right is longer and usually arises slightly higher than the left. It passes posterior to the inferior venacava. The left renal artery arises a little lower down and passes behind the left renal vein. The renal artery enters and the renal vein leaves the kidney on each side through the hilum. Normally one renal artery supplies each kidney and one renal vein drains the kidney. However, variations of the renal arteries are not unusual.

The most commonly observed variation is the presence of an accessory renal artery, occurring in approximately $30 \%$ of cases, arising from the aorta above or below the main renal artery. The accessory renal artery variations have been explained in the

Correspondence : R. Guha.

E-Mail: guharanjit@yahoo.co.in light of embryological development from the lateral mesonephric branches of the dorsal aorta. ${ }^{1}$

The knowledge of variations of renal arteries is helpful for transplantation surgeons, urologists during urological procedures; for vascular surgeons during repair of abdominal aorta aneurysm and for radiologists during angiographic interventions.

\section{Case report}

A case of unilateral accessory renal arteries was found during routine dissection of an adult male cadaver, Department of Anatomy, College of Medical Sciences, Nepal. The abdominal cavity of male cadaver was opened, the small and large intestines were tied and removed. After fine dissection all paired and unpaired branches of abdominal aorta were traced and structurers in the posterior abdominal wall were also observed. We observed that an accessory left inferior renal artery 
reaching the lower pole of left kidney.The accessory renal artery was seen to be passing over the pelvic part of ureter and enters in to the lower pole of left kidney. The main left renal artery passed behind the inferior venacava and reached the upper pole of left kidney.

The artery gave three branches; one large branch passed in front and entered the kidney at its upper pole and the last large branch running posteriorly forming a sulcus along with the vein and supplying the region by giving smaller branches. At the hilum near pelvis, one small branch entered the substance of kidney.(Figure 1,2) No other anomalies were observed in the posterior abdominal wall.

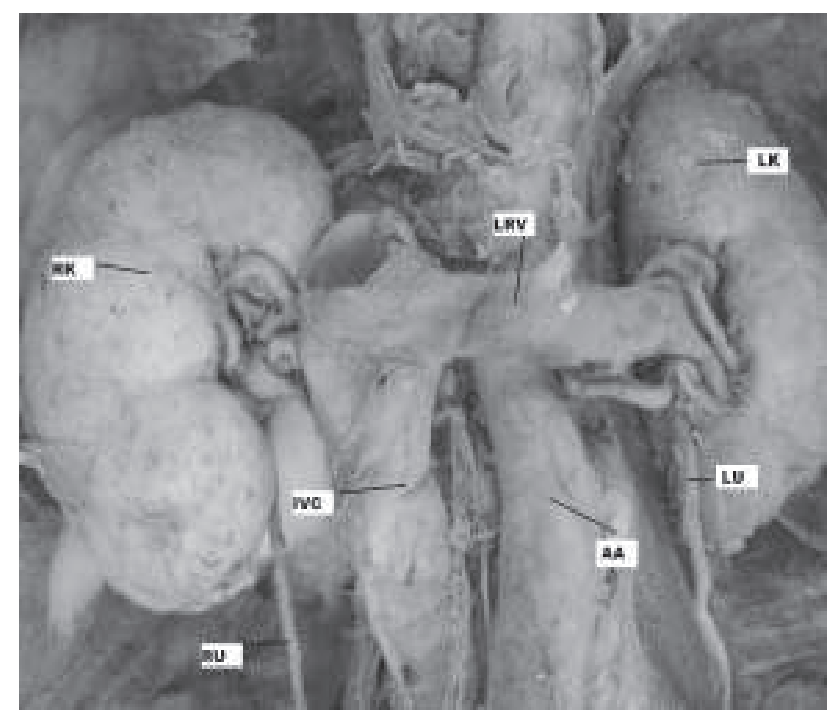

Figure 1. Showing the Right kidney, left kidney, veins and arteries in this area.

RK : Right kidney;LK :Left kidney; IVC: Inferior vena cava; LRV: left renal vein; AA: Abdominal aorta; RU:Right ureter;LU:Left ureter.

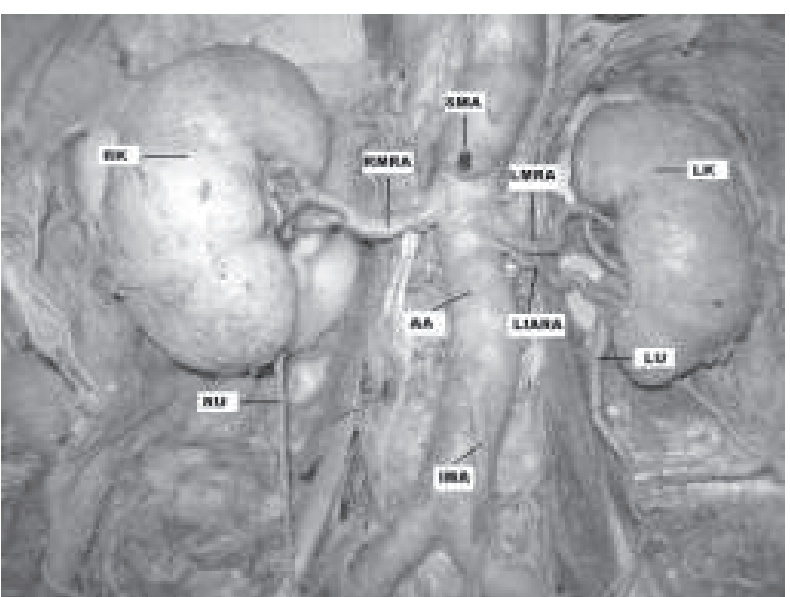

Figure 2. Showing the right kidney,left kidney and the main renal arteries and left accessory renal arteries arising from abdominal aorta. (After removal of inferior venacava, right and left renal veins)

RK: Right kidney; LK: Left kidney; RMRA: Right main renal artery ;LMRA: Left main renal artery; LIARA: Left inferior accessory renal artery; SMA:Superior mesenteric artery; IMA:Inferior mesenteric artery; AA: Abdominal aorta; RU:Right ureter; LU:Left ureter.

\section{Discussion}

Presence of accessory renal arteries can be explained in the light of embryogenic development and its molecular regulation.

Each primitive dorsal aorta gives off ventral splanchnic arteries, lateral splanchenic arteries, somatic arteries and caudal continuation.

The latral splanchnic arteries supply, on each side, the mesonephros, metanephros, the testis or ovary and the suprarenal gland. All these structures develop, in whole or in part, from the intermidiate mesnchyme of the mesonephric ridge. One testicular or ovarian artery and three suparenal arteries persist on each side. The phrenic artery branches from the most cranial suprarenal artery 
N. Satyanarayana et al. Left inferior accessory renal arteries its embryological basis.

frequently present and may be looked on as branches of persistent lateral splanchnic arteries. ${ }^{1}$

Blood vessels develop in two ways: by vasculogenesis, in which vessels arises from blood islands, and by angiogenesis, in which new vessels sprout from existing ones. During vasculogenesis, fibroblast growth factors 2 (FGF2) binds to its receptor on subpopulations of mesoderm cells and induces them to from hemangioblasts. Then, under influence of vascular endothelial growth factor (VEGF) acting through two receptors (VEGF-R2, VEGF-R1), these cells become endothelial and coalesce to form vessels. VEGF also regulates angiogenesis where it stimulates proliferation of endothelial cells at points where new vessels will sprout from existing ones. Final modeling and stabilization of the vasculature are accomplished by platelet-derived growth factor $\hat{\mathrm{a}}_{2}{ }^{2}$

Persistence of lateral splanchnic arteries with branches in the form of accessory renal arteries may be attributed to misexpression of any of these transcription factors and signalling molecules as mentioned above viz VEGF, TGFb, PDGF, SHH and $\mathrm{EphB}_{2}$.

Accessory renal arteries are found frequently on the left side and occurrence is as high as $30-35 \%$ of cases, these arteries usually enter the upper or lower poles of the kidney. ${ }^{3}$

There was much discrepancy regarding the side of accessory renal arteries, many authors have reported a higher frequency on the left side others reported this variation to be more frequent on the right side. ${ }^{4,5}$ In the present study, we found accessory left renal artery on left side entering the lower pole as inferior polar artery. On the right side normal renal artery was found.

Bordei P et al. (2004) reported 54 cases, out of which six cases were bilateral. In about 28 cases, supplementary renal artery entered the kidney through the hilum, in 16 cases it was inferior polar, in five cases it was superior polar. ${ }^{6}$

Incidence of multiple accessory renal arteries has been reported to be $20.2 \%$ and $19 \%$ on right and left sides, respectively by Janschek EC et al.(2004). ${ }^{7}$

Saldarriaga B et al. (2008) reported 97 out of 390 (24.9\%) cases, with kidneys having additional arteries; out of these 87 (22.3\%) had one additional artery and $10(2.6 \%)$ had two additional arteries. The frequency of one additional artery was $43.5 \%$ on right side and $56.3 \%$ on left side. ${ }^{8}$

Incidence of reported additional renal arteries has a wide range between $8.7 \%$ and $75.7 \%$, and they can cause hydronephrosis by compressing the ureter. $^{9}$

This accessory renal arteries is important in surgical procedures related to the posterior abdominal wall, mainly during kidney transplantations, repair of abdominal aortic aneurysm, ureter surgery and surgeries involving the vascular pedicles of kidney. It may also be of practical importance for radiologists, for correct interpretation of roentgenographic examinations in angiographic procedure.

Finally anatomical knowledge of variations of renal arteries is essential before performing any transplantation surgeries, where microvascular techniques are employed to reconstruct the renal arteries. $^{10}$ 


\section{References}

1. S.Standring, H.Ellis, J.C.Healy et al. Gray's Anatomy. $39^{\text {th }}$ ed. Philadelphia Elsevier Churchill Livingstone, 2005;1274,1042.

2. T.W.Saddler. Langman's Medical Embryology. In: blood and blood vessels. 10th ed. Philadelphia Lippincott Williams \& Wilkins, 2006;78.

3. G.Singh, B.H.Bay. Bilateral accessory renal arteries associated with some anomalies of the ovarian arteries:a case study, Clin Anat 1998; 11:417-20.

4. K.S.Satyapal, A.A Haffejee, B.Singh et al. Additional renal arteries incidence and morphometry, Surg Radiol Anat 2000; 23:33-8.

5. S.Nayak. Common celiaco-mesenterico-phrenic trunk and renal vascular variations, Saudi Med J 2006;27 :1894-6.
6. P.Bordei, E.Sapte, D.Iliescu et al. The morphology and the surgical importance of the gonadal arteries originating from renal artery, Surg Radiol Anat, 2007; 29:367-71.

7. E.C.Janschek, A.U.Rothe, T.J.Holzenbein et al. Anatomic basis of right renal vein extension for cadaveric kidney transplantation, J Urology, 2004; 63:660-4.

8. B.Saldarriaga, A.F.Perez, L.E.Ballesteros et al. A direct anatomical study of additional renal arteries in a Colombian mestizo population, Folia Morph 2008; 67:129-34.

9. K.L.Moore, A.F.Dalley. Clinically Oriented Anatomy. 4th ed. Philadelphia: Lippincott Williams and Wilkins, 1999; 202-08, 279-89.

10. G.E.Brannen, W.H.Bush, R.J.Correa et al. Microvascularanagement of multiple renalarteries in transplantation, J Urol 1982; 128:112-5. 